tierte sich die stärkere präventive Wirksamkeit von Clopidogrel auf kardiovaskuläre Ereignisse vor allem in der Subgruppe der Studienteilnehmer mit PAVK.

\section{EUCLID soll Datenlage verbessern}

Vor diesem Hintergrund sollte mit der randomisierten doppelblinden Studie EUCLID die wissenschaftliche Datenlage wesentlich verbessert werden. In diese Studie sind 13.885 Patienten mit symptomatischer PAVK - darunter auch 623 Patienten an deutschen Zentren - aufgenommen worden.

Von den Teilnehmern erfüllten $43 \%$ aufgrund eines erniedrigten KnöchelArm-Indexes (ABI niedriger als 0,80) und $57 \%$ aufgrund vorausgegangener Revaskularisationen die Einschlusskriterien. Rund drei Viertel aller Patienten litten unter Claudicatio-Beschwerden, bei $4,6 \%$ bestand bereits eine kritische Extremitätenischämie.

Die Studienteilnehmer wurden per Randomiserung zwei Gruppen zugeteilt und entweder mit Ticagrelor $(90 \mathrm{mg}$ zweimal täglich) oder Clopidogrel (75 mg einmal täglich) behandelt. Diemediane Follow-up-Dauer betrug 30 Monate.

Ausgehend von der PLATO-Studie, in der Ticagrelor bei Patienten mit akutem Koronarsyndrom (STEMI oder NSTE$\mathrm{MI}$ ) eine überlegene Wirkung im Vergleich zu Clopidogrel offenbart hatte, schien die Erwartung legitim zu sein, dass sich diese Überlegenheit auch bei Patienten mit PAVK belegen lassen würde. Dazu kam es jedoch nicht.

\section{Kein signifikanter Unterschied}

Am Ende konnte kein nennenswerter Unterschied zwischen beiden Plättchenhemmern in ihrer Wirkung auf den primären Endpunkt nachgewiesen werden. Dieser Endpunkt war, wie schon in der PLATO-Studie, als Kombination der Ereignisse kardiovaskulärer Tod, Myokardinfarkt und ischämischer Schlaganfall definiert; die entsprechenden Ereignisraten waren mit 10,8\% (Ticagrelor) und 10,6\% (Clopidogrel) nicht signifikant unterschiedlich. Beim Einzelendpunkt ischämischer Schlaganfall fiel das Ergebnis allerdings signifikant zugunsten von Ticagrelor aus (1,9 vs. 2,4\%).

Die Inzidenzraten für akute Extremitätenischämien waren in beiden Behandlungsgruppen mit jeweils 1,7\% identisch. Auch mit Blick auf die Rate schwerwiegender Blutungskomplikationen (jeweils $1,6 \%$ ) bestand Gleichheit zwischen beiden Thrombozytenhemmern.

Allerdings war im Ticagrelor-Arm eine höhere Rate an vorzeitigen Therapieabbrüchen zu verzeichnen (30,1 vs. $25,9 \%)$. Dieser Unterschied ist primär dem häufigeren Auftreten von Dyspnoen und von leichten Blutungen unter Ticagrelor geschuldet.
Warum diesmal keine Überlegenheit?

Warum hat sich die bei akutem Koronarsyndrom belegte Überlegenheit von $\mathrm{Ti}$ cagrelor gegenüber Clopidogrel bei PAVK nicht nachweisen lassen? Ein Grund könnten Unterschiede in der Pathophysiologie sein. Das akute Koronarsyndrom ist im Unterschied zur PAVK durch instabile Koronarläsionen in Verbindung mit einer deutlich gesteigerten Plättchenaggregabilität gekennzeichnet. Deshalb könnte hier die stärkere plättchenhemmende Wirkung von Ticagrelor klinisch stärker zum Tragen kommen.

Clopidogrel ist bekanntlich ein Prodrug. Erst nach Resorption entsteht durch Oxidation und anschließende Hydrolyse der pharmakologisch aktive Metabolit. Bei vielen Menschen ist die Metabolisierung und damit die Wirksamkeit von Clopidogrel genetisch bedingt eingeschränkt („poor metabolizer“). Als „poor metabolizer" identifizierte Patienten waren von der EUCLID-Studie - anders als bei früheren Studien - ausgeschlossen. Dadurch könnte die Wirksamkeit von Clopidogrel der von Ticagrelor angeglichen worden sein.

Peter Overbeck

Quelle: Präsentation in der Sitzung „Late-Breaking Clinical Trials 01 - Big Trials for Big Questions" am 13.11.2016 beim Kongress der American Heart Association (AHA) 2016, 12.-16.11.2016, New Orleans

Hiatt WR et al. Ticagrelor versus Clopidogrel in Symptomatic Peripheral Artery Disease. N Engl J Med, 2016 Nov 13; doi: 10.1056/NEJMoa1611688

\title{
Erhöhtes Herztod-Risiko nach Jojo-Effekt
}

Postmenopausale Frauen, die wiederholt ihr Gewicht reduzieren, aber den Erfolg nicht halten, könnten ein erhöhtes Herztodrisiko aufweisen - aber nur, wenn sie normalgewichtig sind.

Autoren fanden ein um den Faktor 3,5 erhöhtes Risiko für einen plötzlichen Herztod sowie ein um den Faktor 1,66 erhöhtes Risiko für eine koronare Herzkrankheit bei postmenopausalen Frauen mit wiederholten Gewichtsschwankungen.

Die Risiken waren aber nur bei solchen Frauen erhöht, die zu Beginn normalgewichtig waren. Bei übergewichtigen oder gar adipösen Frauen bargen Diäten mit Jojo-Effekt keine kardialen Risiken, berichtete Studienautor Dr. Somwail Rasla von der Brown University in Providence, Rhode Island.

Auch wenn Frauen an Gewicht zulegten, ohne es wieder zu verlieren, war das kardiale Risiko nicht erhöht. Ebenso wenig bei Frauen, die Gewicht verloren und das niedrigere Gewicht beibehielten.

Die Autoren hatten bei einem Kollektiv von 158.063 postmenopausen Frauen den Gewichtsverlauf über 11,4 Jahre beobachtet und sie in die Gruppen „konstantes Gewicht" , „allmähliche Gewichtszunahme”, "Gewichtsabnahme" und "Gewichtsschwankungen" eingeteilt.

Die Studie hat Schwächen. Zum einen ist es eine Beobachtungsstudie, zum anderen war man auf die Angaben der Teilnehmerinnen angewiesen, zum dritten wurden nur ältere Frauen erfasst. 